\title{
HYPERGEOMETRIC FUNCTIONS OVER FINITE FIELDS
}

\author{
JOHN GREENE
}

\begin{abstract}
In this paper the analogy between the character sum expansion of a complex-valued function over $\operatorname{GF}(q)$ and the power series expansion of an analytic function is exploited in order to develop an analogue for hypergeometric series over finite fields. It is shown that such functions satisfy many summation and transformation formulas analogous to their classical counterparts.
\end{abstract}

1. Introduction and notation. Let $\mathrm{GF}(q)$ denote the finite field with $q$ elements where $q$ is a power of a prime. If $\chi$ is a multiplicative character of $\operatorname{GF}(q)^{*}$, extend $\chi$ to all of $\operatorname{GF}(q)$ by setting $\chi(0)=0$. It is easy to see that any function $f$ : $\mathrm{GF}(q) \rightarrow \mathbf{C}$ has a unique representation

$$
f(x)=f_{\delta} \delta(x)+\sum_{\chi} f_{\chi} \chi(x)
$$

where the sum ranges over all multiplicative characters of $\operatorname{GF}(q)$ and $\delta$ is the function

$$
\delta(x)= \begin{cases}1 & \text { if } x=0 \\ 0 & \text { if } x \neq 0\end{cases}
$$

In fact, the constants $f_{\delta}$ and $f_{\chi}$ are given by

$$
f_{\delta}=f(0), \quad f_{\chi}=\frac{1}{q-1} \sum_{x \in \mathrm{GF}(q)} f(x) \bar{\chi}(x) .
$$

The expression (1.1) represents a finite field analogue for the power series expansion of a function.

As an example, let $\operatorname{Tr}$ be the trace map from $\operatorname{GF}(q)$ to $\operatorname{GF}(p)$, set $\zeta=e^{2 \pi i / p}$ and define the Gauss sum by

$$
G(\chi)=\sum_{t \in \mathrm{GF}(q)} \chi(t) \zeta^{\operatorname{Tr}(t)}
$$

The Gauss sum is the analogue of the gamma function

$$
\Gamma(x)=\int_{0}^{\infty} t^{x} e^{-t} \frac{d t}{t} .
$$

Received by the editors January 16, 1986.

1980 Mathematics Subject Classification (1985 Revision). Primary 11T21, 11Lxx; Secondary 33A30, $11 \mathrm{G} 20$.

This paper contains parts of the author's Ph.D. thesis written under the supervision of Professor D. Stanton at the University of Minnesota. 
The power series expansion for $e^{x}$ can be written in terms of the gamma function

$$
e^{x}=\sum_{k=0}^{\infty} \frac{x^{k}}{\Gamma(k+1)} .
$$

Comparing (1.4) and (1.5) one might guess that an analogous formula holds for $\zeta^{-\operatorname{Tr}(x)}$ and, in fact, it follows easily from (1.3) that

$$
\zeta^{-\operatorname{Tr}(x)}=1+\frac{q}{q-1} \sum_{\chi} \frac{\chi(x)}{G(\chi)} .
$$

This analogy, which can essentially be expressed by $x^{k} \leftrightarrow \chi(x)$, dates back at least to Jacobi (see for example [13]). Recently there has been renewed interest in this analogy. For example, in [11, Theorem 2] Helversen-Pasotto gives a result

$$
\begin{aligned}
& \frac{1}{q-1} \sum_{\chi} G(A \chi) G(B \bar{\chi}) G(C \chi) G(D \bar{\chi}) \\
& \quad=\frac{G(A B) G(A D) G(B C) G(C D)}{G(A B C D)}+q(q-1) A C(-1) \delta(A B C D),
\end{aligned}
$$

where the term $\delta(A B C D)$ is 1 if $A B C D$ is the trivial character and 0 otherwise. This result is analogous to Barnes' lemma

$$
\begin{aligned}
\frac{1}{2 \pi i} \int_{-i \infty}^{i \infty} \Gamma(a+z) \Gamma & (b-z) \Gamma(c+z) \Gamma(d-z) d z \\
= & \frac{\Gamma(a+b) \Gamma(a+d) \Gamma(b+c) \Gamma(c+d)}{\Gamma(a+b+c+d)}
\end{aligned}
$$

where the path of integration is curved so as to separate the increasing and decreasing sequence of poles.

More examples of this analogy can be found in the references [5-11, 14 and 15]. Evans [7] has recently exploited this analogy to produce character sum analogues for Hermite polynomials and has shown that many of the classical properties carry over to the finite field case. In this paper we conduct a similar study of a character sum analogue for hypergeometric series. It is hoped that a study of special function analogues will serve as a useful tool when applied to problems surrounding character sums in the literature.

The organization of this paper is as follows. In $\$ 2$ we derive an analogue for the binomial theorem. Binomial coefficients are then defined and their elementary properties are investigated. In $\$ 3$, hypergeometric and generalized hypergeometric series are introduced. Transformations and summation theorems are given in $\S 4$ and we give examples in $\S 5$. In the remainder of this section we set notation.

Throughout this paper, capital letters $A, B, C, \ldots$ and Greek letters $\chi, \psi, \ldots$ will denote multiplicative characters. We will let $\delta$ denote both the function on $\operatorname{GF}(q)$ defined in (1.2) and the function on characters

$$
\delta(A)= \begin{cases}1 & \text { if } A \text { is the trivial character } \\ 0 & \text { otherwise. }\end{cases}
$$


The trivial character will be denoted $\varepsilon$ and the quadratic character $\varphi$. Define $\bar{\chi}$ by $\chi \bar{\chi}=\varepsilon$. Write $\sum_{x}$ to denote the sum over all $x$ in $\mathrm{GF}(q)$ and $\sum_{x}$ to denote the sum over all characters of $\operatorname{GF}(q)$. Let $J(A, B)$ denote the Jacobi sum

$$
J(A, B)=\sum_{x} A(x) B(1-x) .
$$

Elementary properties of Gauss and Jacobi sums which we will make use of are [12, pp. 92, 93]

$$
\begin{gathered}
G(A) G(\bar{A})=q A(-1)-(q-1) \delta(A)=q A(-1)\left(1-\frac{q-1}{q} \delta(A)\right) \\
J(A, B)=J(B, A)
\end{gathered}
$$

and

$$
J(A, B)=\frac{G(A) G(B)}{G(A B)}+(q-1) B(-1) \delta(A B) .
$$

We will also make frequent use of the orthogonality relations for characters [12, pp. $89,90]$

$$
\sum_{x} A(x) B(x)=(q-1) \delta(A B)
$$

and

$$
\sum_{\chi} \chi(x)=(q-1) \delta(1-x)
$$

2. Binomial coefficients and the binomial theorem. The binomial theorem states

$$
(1+x)^{a}=\sum_{k=0}^{\infty}\left(\begin{array}{l}
a \\
k
\end{array}\right) x^{k}
$$

where $\left(\begin{array}{l}a \\ k\end{array}\right)$ is the "binomial coefficient" defined by

$$
\left(\begin{array}{l}
a \\
k
\end{array}\right)=\frac{a(a-1) \cdots(a-k+1)}{k !}=\frac{\Gamma(a+1)}{\Gamma(k+1) \Gamma(a-k+1)} .
$$

A character sum analogue for (2.1) follows easily from (1.1) with $f(x)=A(1+x)$. The result is

THEOREM 2.3. For any character $A$ of $\mathrm{GF}(q)$ and $x \in \mathrm{GF}(q)$,

$$
A(1+x)=\delta(x)+\frac{1}{q-1} \sum_{\chi} J(A, \bar{\chi}) \chi(-x) .
$$

Proof. By (1.3),

$$
f_{\chi}=\frac{1}{q-1} \sum_{x} A(1+x) \bar{\chi}(x) .
$$

The result follows from the change of variables $y=-(1-x)$.

It follows from Theorem 2.3 that the finite field analogue for the binomial coefficient is the Jacobi sum. It will prove useful to make this analogy more explicit. 
Definition 2.4. For characters $A$ and $B$ of $\operatorname{GF}(q)$ define $\left({ }_{B}^{A}\right)$ by

$$
\left(\begin{array}{l}
A \\
B
\end{array}\right)=\frac{B(-1)}{q} J(A, \bar{B}) .
$$

In terms of binomial coefficients, Theorem 2.3 can be written

$$
A(1+x)=\delta(x)+\frac{q}{q-1} \sum_{\chi}\left(\begin{array}{l}
A \\
\chi
\end{array}\right) \chi(x) .
$$

Some useful properties of binomial coefficients which follow easily from properties of Jacobi sums are

$$
\begin{gathered}
\left(\begin{array}{l}
A \\
B
\end{array}\right)=\left(\begin{array}{c}
A \\
A \bar{B}
\end{array}\right), \\
\left(\begin{array}{l}
A \\
B
\end{array}\right)=\left(\begin{array}{c}
B \bar{A} \\
B
\end{array}\right) B(-1), \\
\left(\begin{array}{l}
A \\
B
\end{array}\right)=\left(\begin{array}{c}
\bar{B} \\
\bar{A}
\end{array}\right) A B(-1),
\end{gathered}
$$

and

$$
\begin{aligned}
\left(\begin{array}{l}
A \\
B
\end{array}\right) & =\frac{G(A) G(\bar{B}) B(-1)}{G(A \bar{B}) q}+\frac{q-1}{q} \delta(A \bar{B}) \\
& =\frac{G(A)}{G(B) G(A \bar{B})}+\frac{q-1}{q} \delta(B)+\frac{q-1}{q} \delta(A \bar{B}) .
\end{aligned}
$$

Some important variants of the binomial theorem are

$$
\bar{A}(1-x)=\delta(x)+\frac{q}{q-1} \sum_{\chi}\left(\begin{array}{c}
A \chi \\
\chi
\end{array}\right) \chi(x)
$$

and

$$
\bar{B}(x) \bar{A} B(1-x)=\frac{q}{q-1} \sum_{\chi}\left(\begin{array}{l}
A \chi \\
B \chi
\end{array}\right) \chi(x) .
$$

The result in (2.10) follows from (2.5) and (2.7). The result in (2.11) follows from (2.10) and the change of variables $\chi \mapsto \bar{B} \chi$.

From (2.9) we derive the following special cases for binomial coefficients

$$
\begin{gathered}
\left(\begin{array}{c}
A \\
\varepsilon
\end{array}\right)=\left(\begin{array}{l}
A \\
A
\end{array}\right)=-\frac{1}{q}+\frac{q-1}{q} \delta(A), \\
\left(\begin{array}{c}
\varepsilon \\
A
\end{array}\right)=-\frac{A(-1)}{q}+\frac{q-1}{q} \delta(A)
\end{gathered}
$$

and

$$
\left(\begin{array}{l}
\varepsilon \\
\varepsilon
\end{array}\right)=\frac{q-2}{q}
$$


Less trivial but very important are the formulas

$$
\begin{aligned}
\left(\begin{array}{l}
A \\
B
\end{array}\right)\left(\begin{array}{l}
C \\
A
\end{array}\right)= & \left(\begin{array}{l}
C \\
B
\end{array}\right)\left(\begin{array}{l}
C \bar{B} \\
A \bar{B}
\end{array}\right)-\frac{q-1}{q^{2}} B(-1) \delta(A) \\
& +\frac{q-1}{q^{2}} A B(-1) \delta(B \bar{C})
\end{aligned}
$$

and

$$
\left(\begin{array}{c}
A^{2} \\
A B
\end{array}\right)\left(\begin{array}{c}
\varphi \\
B
\end{array}\right)=\left(\begin{array}{c}
A \\
B
\end{array}\right)\left(\begin{array}{c}
\varphi A \\
A B
\end{array}\right) A(4)
$$

The result in (2.15) is the analogue of the well-known classical identity for binomial coefficients

$$
\left(\begin{array}{l}
n \\
k
\end{array}\right)\left(\begin{array}{c}
m \\
n
\end{array}\right)=\left(\begin{array}{c}
m \\
k
\end{array}\right)\left(\begin{array}{c}
m-k \\
n-k
\end{array}\right)
$$

The proof of (2.15) follows from a tedious but straightforward calculation using (2.9). The result in (2.16) is similar except that one must also invoke a special case of the Hasse-Davenport relation [3, p. 153]

$$
G(A) G(\varphi A)=G\left(A^{2}\right) G(\varphi) \bar{A}(4) .
$$

3. Hypergeometric series. Recall that the hypergeometric series is defined by

$$
{ }_{2} F_{1}(a, \quad b \mid x)=\sum_{k=0}^{\infty} \frac{(a)_{k}(b)_{k}}{k !(c)_{k}} x^{k},
$$

where

$$
(a)_{k}=\frac{\Gamma(a+k)}{\Gamma(a)}=a(a+1) \cdots(a+k-1) .
$$

More generally,

$$
{ }_{r} F_{s}\left(\begin{array}{c}
a_{1}, a_{2}, \ldots, a_{r} \\
b_{1}, b_{2}, \ldots, b_{s}
\end{array} \mid x\right)=\sum_{k=0}^{\infty} \frac{\left(a_{1}\right)_{k}\left(a_{2}\right)_{k} \cdots\left(a_{r}\right)_{k}}{k !\left(b_{1}\right)_{k} \cdots\left(b_{s}\right)_{k}} x^{k} .
$$

The most elementary attempt to define a character sum analogue for (3.3) would thus be

$$
\frac{G\left(B_{1}\right) G\left(B_{2}\right) \cdots G\left(B_{s}\right)}{G\left(A_{1}\right) G\left(A_{2}\right) \cdots G\left(A_{r}\right)} \sum_{\chi} \frac{G\left(A_{1} \chi\right) G\left(A_{2} \chi\right) \cdots G\left(A_{r} \chi\right)}{G(\chi) G\left(B_{1} \chi\right) \cdots G\left(B_{s} \chi\right)} \chi(x),
$$

using $G(A \chi) / G(A)$ as an analogue for $\Gamma(a+k) / \Gamma(a)$. Unfortunately, this approach leads to rather poor results. An alternative that gives better results is to develop an analogue for the integral representation for the hypergeometric series [ 2 , p. 4]

(3.4) ${ }_{2} F_{1}(a, \quad b \mid x)=\frac{\Gamma(c)}{\Gamma(b) \Gamma(c-b)} \int_{0}^{1} t^{b}(1-t)^{c-b}(1-t x)^{-a} \frac{d t}{t(1-t)}$. 
Definition 3.5. For characters $A, B$ and $C$ of $\mathrm{GF}(q)$ and $x \in \mathrm{GF}(q)$,

$$
{ }_{2} F_{1}\left(\begin{array}{ll}
A, & B \\
& C
\end{array} \mid x\right)=\varepsilon(x) \frac{B C(-1)}{q} \sum_{y} B(y) \bar{B} C(1-y) \bar{A}(1-x y) .
$$

In this definition we have dropped the normalizing constant $\Gamma(c) / \Gamma(b) \Gamma(c-b)$ in order to obtain simpler results. The factor $\varepsilon(x) B C(-1) / q$ is chosen so as to lead to a better expression in terms of binomial coefficients.

Theorem 3.6. For characters $A, B$ and $C$ of $\mathrm{GF}(q)$ and $x \in \mathrm{GF}(q)$,

$$
{ }_{2} F_{1}\left(\begin{array}{cc}
A, & B \\
& C
\end{array} \mid x\right)=\frac{q}{q-1} \sum_{\chi}\left(\begin{array}{r}
A \chi \\
\chi
\end{array}\right)\left(\begin{array}{l}
B \chi \\
C \chi
\end{array}\right) \chi(x) .
$$

Proof. By (2.10),

$$
\bar{A}(1-x y)=\delta(x y)+\frac{q}{q-1} \sum_{\chi}\left(\begin{array}{r}
A \chi \\
\chi
\end{array}\right) \chi(x y) .
$$

Since $\varepsilon(x) B(y) \delta(x y)=0$ for all $x, y$ we have

$$
\begin{aligned}
{ }_{2} F_{1}\left(\begin{array}{rl}
A, & B \\
C
\end{array} \mid x\right) & =\frac{B C(-1)}{q-1} \sum_{y, \chi}\left(\begin{array}{r}
A \chi \\
\chi
\end{array}\right) \chi(x) B \chi(y) \bar{B} C(1-y) \\
& =\frac{B C(-1)}{q-1} \sum_{\chi}\left(\begin{array}{r}
A \chi \\
\chi
\end{array}\right) J(B \chi, \bar{B} C) \chi(x) \\
& =\frac{q}{q-1} \sum_{\chi}\left(\begin{array}{r}
A \chi \\
\chi
\end{array}\right)\left(\begin{array}{r}
B \chi \\
B \bar{C}
\end{array}\right) \chi(x) .
\end{aligned}
$$

The result now follows since

$$
\left(\begin{array}{l}
B \chi \\
B \bar{C}
\end{array}\right)=\left(\begin{array}{l}
B \chi \\
C \chi
\end{array}\right)
$$

Note that the classical hypergeometric series can also be expressed in terms of binomial coefficients

$$
{ }_{2} F_{1}\left(\begin{array}{ll}
a, & b \\
c
\end{array} \mid x\right)=C \sum_{k=0}^{\infty}\left(\begin{array}{c}
a+k-1 \\
k
\end{array}\right)\left(\begin{array}{l}
b+k-1 \\
c+k-1
\end{array}\right) x^{k} .
$$

More generally,

$$
{ }_{n+1} F_{n}\left(\begin{array}{c}
a_{0}, a_{1}, \ldots, a_{n} \\
b_{1}, \ldots, b_{n}
\end{array} \mid x\right)=C \sum_{k=0}^{\infty}\left(\begin{array}{c}
a_{0}+k-1 \\
k
\end{array}\right)\left(\begin{array}{c}
a_{1}+k-1 \\
b_{1}+k-1
\end{array}\right) \cdots\left(\begin{array}{c}
a_{n}+k-1 \\
b_{n}+k-1
\end{array}\right) x^{k}
$$

where

$$
C=\left\{\left(\begin{array}{l}
a_{1}-1 \\
b_{1}-1
\end{array}\right) \cdots\left(\begin{array}{l}
a_{n}-1 \\
b_{n}-1
\end{array}\right)\right\}^{-1} .
$$

This leads directly to the following definition. 
Definition 3.10. For characters $A_{0}, A_{1}, \ldots, A_{n}, B_{1}, \ldots, B_{n}$ of $\operatorname{GF}(q)$ and $x \in$ $\mathrm{GF}(q)$,

$$
{ }_{n+1} F_{n}\left(\begin{array}{r}
A_{0}, A_{1}, \ldots, A_{n} \\
B_{1}, \ldots, B_{n}
\end{array} \mid \chi\right)=\frac{q}{q-1} \sum_{\chi}\left(\begin{array}{c}
A_{0} \chi \\
\chi
\end{array}\right)\left(\begin{array}{c}
A_{1} \chi \\
B_{1} \chi
\end{array}\right) \cdots\left(\begin{array}{c}
A_{n} \chi \\
B_{n} \chi
\end{array}\right) \chi(x) .
$$

Again we drop the factor $C$ in order to obtain simpler results later on. The important special case

$$
{ }_{1} F_{0}(A \mid x)=\varepsilon(x) \bar{A}(1-x)
$$

follows from (2.10).

Generalized hypergeometric series have the following inductive integral representation [1, p. 19]

$$
\begin{aligned}
{ }_{n+1} F_{n}\left(\begin{array}{r}
a_{0}, a_{1}, \ldots, a_{n} \\
b_{1}, \ldots, b_{n}
\end{array} \mid x\right)= & \frac{\Gamma\left(b_{n}\right)}{\Gamma\left(a_{n}\right) \Gamma\left(b_{n}-a_{n}\right)} \int_{0}^{1}{ }_{n} F_{n-1}\left(\begin{array}{r}
a_{0}, a_{1}, \ldots, a_{n-1} \\
b_{1}, \ldots, b_{n-1}
\end{array} \mid t x\right) \\
& \cdot t^{a_{n}}(1-t)^{b_{n}-a_{n}} \frac{d t}{t(1-t)} .
\end{aligned}
$$

The analogue for this result also holds.

Theorem 3.13. For characters $A_{0}, A_{1}, \ldots, A_{n}, B_{1}, \ldots, B_{n}$ of $\mathrm{GF}(q)$ and $x \in$ $\mathrm{GF}(q)$,

$$
\begin{aligned}
{ }_{n+1} F_{n}\left(\begin{array}{r}
A_{0}, A_{1}, \ldots, A_{n} \\
B_{1}, \ldots, B_{n}
\end{array} \mid x\right)= & \frac{A_{n} B_{n}(-1)}{q} \sum_{y} F_{n-1}\left(\begin{array}{r}
A_{0}, A_{1}, \ldots, A_{n-1} \\
B_{1}, \ldots, B_{n-1}
\end{array} \mid x y\right) \\
& \cdot A_{n}(y) \overline{A_{n}} B_{n}(1-y) .
\end{aligned}
$$

Proof. We use

$$
\begin{aligned}
\left(\begin{array}{c}
A_{n} \chi \\
B_{n} \chi
\end{array}\right) & =\left(\begin{array}{c}
A_{n} \chi \\
A_{n} \bar{B}_{n}
\end{array}\right)=\frac{A_{n} B_{n}(-1)}{q} J\left(A_{n} \chi, \overline{A_{n}} B_{n}\right) \\
& =\frac{A_{n} B_{n}(-1)}{q} \sum_{y} A_{n} \chi(y) \overline{A_{n}} B_{n}(1-y)
\end{aligned}
$$

in Definition 3.10 to obtain

$$
\begin{aligned}
{ }_{n+1} F_{n}\left(\begin{array}{r}
A_{0}, A_{1}, \ldots, A_{n} \\
B_{1}, \ldots, B_{n}
\end{array} \mid x\right)= & \frac{A_{n} B_{n}(-1)}{q} \sum_{y} \frac{q}{q-1} \sum_{\chi}\left(\begin{array}{c}
A_{0} \chi \\
\chi
\end{array}\right) \cdots\left(\begin{array}{c}
A_{n-1} \chi \\
B_{n-1} \chi
\end{array}\right) \\
& \cdot \chi(x y) A_{n}(y) \overline{A_{n}} B_{n}(1-y),
\end{aligned}
$$

and the result follows.

COROLlary 3.14. For characters $A, B, C, D$ and $E$ of $\mathrm{GF}(q)$ and $x \in \mathrm{GF}(q)$,

(i) ${ }_{3} F_{2}\left(\begin{array}{c}A, B, C \\ D, E\end{array} \mid x\right)$

$$
=\varepsilon(x) \frac{B C D E(-1)}{q^{2}} \sum_{y, z} C(y) \bar{C} E(1-y) B(z) \bar{B} D(1-z) \bar{A}(1-x y z),
$$


(ii) ${ }_{3} F_{2}\left(\begin{array}{r}A, B, C \\ D, E\end{array} \mid x\right)$

$$
=\varepsilon(x) \frac{B D(-1)}{q^{2}} \sum_{y, z} A \bar{E}(y) \bar{C} E(1-y) B(z) \bar{B} D(1-z) \bar{A}(y-x z) .
$$

Proof. The result in (i) follows from Theorem 3.13 and Definition 3.5. The result in (ii) follows from (i) by the change of variables $y \mapsto 1 / y$.

It is clear from (3.3) that for the classical hypergeometric series, if a numerator parameter equals a denominator parameter, then the series can be written as a hypergeometric series with $r$ and $s$ each reduced by one. We expect a similar result in the finite field case. In addition, the series will also reduce to one of lower order if one of the numerator parameters is the trivial character. To simplify notation we adopt the following conventions: for characters $A_{1}, \ldots, A_{n}, B_{1}, \ldots, B_{n}$ let

$$
\left(\begin{array}{l}
\vec{A} \\
\vec{B}
\end{array}\right)
$$

denote the product

$$
\prod_{k=1}^{n}\left(\begin{array}{c}
A_{k} \\
B_{k}
\end{array}\right)
$$

and let

$$
F\left(\begin{array}{rr}
C, & \vec{A} \\
\vec{B} & x
\end{array}\right)
$$

denote the series

$$
F\left(\begin{array}{r}
C, A_{1}, \ldots, A_{n} \\
B_{1}, \ldots, B_{n}
\end{array} \mid x\right) .
$$

Since the order of the binomial coefficients in (3.10) is irrelevant, there are five cases to consider.

THEOREM 3.15. Using the above notation,

$$
\begin{gathered}
F\left(\begin{array}{r}
\varepsilon, A, \vec{B} \\
C, \vec{D}
\end{array} \mid x\right)=-\frac{1}{q} \bar{C}(x) F\left(\begin{array}{r}
A \bar{C}, \vec{B} \bar{C} \\
\vec{D} \bar{C}
\end{array} \mid x\right)+\varepsilon(x)\left(\begin{array}{l}
A \\
C
\end{array}\right)\left(\begin{array}{l}
\vec{B} \\
\vec{D}
\end{array}\right), \\
F\left(\begin{array}{r}
A, B, \vec{C} \\
B, \vec{D}
\end{array} \mid x\right)=-\frac{1}{q} F\left(\begin{array}{r}
A, \vec{C} \\
\vec{D}
\end{array} \mid x\right)+\bar{B}(x)\left(\begin{array}{r}
A \bar{B} \\
\bar{B}
\end{array}\right)\left(\begin{array}{l}
\vec{C} \bar{B} \\
\vec{D} \bar{B}
\end{array}\right),
\end{gathered}
$$

$$
\begin{aligned}
& F\left(\begin{array}{r}
A, \varepsilon, B, \vec{C} \\
D, E, \vec{F}
\end{array} \mid x\right)=A(-1) \bar{D}(x)\left(\begin{array}{l}
D \\
A
\end{array}\right) F\left(\begin{array}{r}
A \bar{D}, B \bar{D}, \vec{C} \bar{D} \\
E \bar{D}, \vec{F} \bar{D}
\end{array} \mid x\right) \\
& \quad-\frac{1}{q} D(-1) \varepsilon(x)\left(\begin{array}{r}
B \\
E
\end{array}\right)\left(\begin{array}{c}
\vec{C} \\
\vec{F}
\end{array}\right)+\frac{q-1}{q^{2}} D(-1) \bar{E}(x) F\left(\begin{array}{r}
B \bar{E}, \vec{C} \bar{E} \\
\vec{F} \bar{E}
\end{array} \mid x\right) \delta(A \bar{D}),
\end{aligned}
$$


(iv)

$$
\begin{aligned}
F\left(\begin{array}{r}
A, B, C, \vec{D} \\
A, E, \vec{F}
\end{array} \mid x\right)= & \left(\begin{array}{c}
B \\
A
\end{array}\right) F\left(\begin{array}{r}
B, C, \vec{D} \\
E, \vec{F}
\end{array} \mid x\right)-\frac{1}{q} \bar{A}(-x)\left(\begin{array}{l}
C \bar{A} \\
E \bar{A}
\end{array}\right)\left(\begin{array}{l}
\vec{D} \bar{A} \\
\vec{F} \bar{A}
\end{array}\right) \\
& +\frac{q-1}{q^{2}} A(-1) \bar{E}(x) F\left(\begin{array}{r}
C \bar{E}, \vec{D} \bar{E} \\
\vec{F} \bar{E}
\end{array} \mid x\right) \delta(B),
\end{aligned}
$$

(v) $\quad F\left(\begin{array}{r}A, B, C, \vec{D} \\ E, B, \vec{F}\end{array} \mid x\right)=\left(\begin{array}{c}C \bar{E} \\ B \bar{E}\end{array}\right) F\left(\begin{array}{r}A, C, \vec{D} \\ E, \vec{F}\end{array} \mid x\right)-\frac{1}{q} B E(-1) \bar{B}(x)\left(\begin{array}{c}A \bar{B} \\ \bar{B}\end{array}\right)\left(\begin{array}{c}\vec{D} \bar{B} \\ \vec{F} \bar{B}\end{array}\right)$

$$
+\frac{q-1}{q^{2}} B E(-1) F\left(\begin{array}{r}
A, \vec{D} \\
\vec{F}
\end{array} \mid x\right) \delta(C \bar{E}) \text {. }
$$

Proof. Cases (i) and (ii) follow easily from

$$
\left(\begin{array}{l}
A \chi \\
A \chi
\end{array}\right)=-\frac{1}{q}+\frac{q-1}{q} \delta(A \chi)
$$

Cases (iii), (iv) and (v) follow from (2.15). For example, in (iv) the binomial coefficients

$$
\left(\begin{array}{c}
A \chi \\
\chi
\end{array}\right)\left(\begin{array}{c}
B \chi \\
A \chi
\end{array}\right)
$$

appear in the character sum for $F$. By (2.15)

$$
\left(\begin{array}{r}
A \chi \\
\chi
\end{array}\right)\left(\begin{array}{c}
B \chi \\
A \chi
\end{array}\right)=\left(\begin{array}{r}
B \chi \\
\chi
\end{array}\right)\left(\begin{array}{l}
B \\
A
\end{array}\right)-\frac{q-1}{q^{2}} \chi(-1) \delta(A \chi)+\frac{q-1}{q^{2}} A(-1) \delta(B),
$$

and the result follows.

An important special case of Theorem 3.15 is where $n=1$.

Corollary 3.16. For characters $A, B, C$ of $\mathrm{GF}(q)$ and $x \in \mathrm{GF}(q)$,

$$
\begin{aligned}
{ }_{2} F_{1}\left(\begin{array}{r}
\varepsilon, B \\
C
\end{array} \mid x\right)=\left(\begin{array}{c}
B \\
C
\end{array}\right) \varepsilon(x)-\frac{1}{q} \bar{C}(x) \bar{B} C(1-x), \\
{ }_{2} F_{1}\left(\begin{array}{r}
A, \varepsilon \\
C
\end{array} \mid x\right)=\left(\begin{array}{l}
C \\
A
\end{array}\right) A(-1) \bar{C}(x) \bar{A} C(1-x) \\
-\frac{1}{q} C(-1) \varepsilon(x)+\frac{q-1}{q} A(-1) \delta(1-x) \delta(\overline{A C}), \\
{ }_{2} F_{1}\left(\begin{array}{r}
A, B \\
A
\end{array} \mid x\right)=\left(\begin{array}{c}
B \\
A
\end{array}\right) \varepsilon(x) \bar{B}(1-x) \\
-\frac{1}{q} \bar{A}(-x)+\frac{q-1}{q} A(-1) \delta(1-x) \delta(B), \\
{ }_{2} F_{1}\left(\begin{array}{r}
A, B \\
B
\end{array} \mid x\right)=-\frac{1}{q} \varepsilon(x) \bar{A}(1-x)+\left(\begin{array}{l}
B \\
A
\end{array}\right) A(-1) \bar{B}(x) .
\end{aligned}
$$

Definition 3.17. A hypergeometric series to which Theorem 3.15 applies is called degenerate.

Another important topic to be discussed is that of symmetry. The classical hypergeometric function is invariant under any permutation of either the numerator or the denominator parameters. As Theorem 3.15 shows, that is not the case for our 
analogue. There are some obvious symmetries present; for example, numerator and denominator parameters are paired off in binomial coefficients in Definition 3.10 so the series is invariant under permutations of pairs of parameters:

$$
{ }_{3} F_{2}\left(\begin{array}{r}
A, B, C \\
D, E
\end{array} \mid x\right)={ }_{3} F_{2}\left(\begin{array}{r}
A, C, B \\
E, D
\end{array} \mid x\right) .
$$

Similarly, special cases can occur; for example,

$$
{ }_{3} F_{2}\left(\begin{array}{r}
A, B, B \\
C, D
\end{array} \mid x\right)={ }_{3} F_{2}\left(\begin{array}{r}
A, B, B \\
D, C
\end{array} \mid x\right) .
$$

We now derive the relation in general between series if parameters are permuted. We restrict our attention to transpositions. By the symmetry we know to exist, we need only consider two cases.

THEOREM 3.20. In the notation preceding Theorem 3.15,

(i)

$$
\begin{aligned}
\left(\begin{array}{r}
B \bar{D} \\
A \bar{D}
\end{array}\right) F\left(\begin{array}{r}
A, B, \vec{C} \\
D, \vec{E}
\end{array} \mid x\right)-\frac{q-1}{q^{2}}\left(\begin{array}{l}
\vec{C} \\
\vec{E}
\end{array}\right) D(-1) \varepsilon(x) \delta(A) \\
+\frac{q-1}{q^{2}} A D(-1) F\left(\begin{array}{r}
A, \vec{C} \\
\vec{E}
\end{array} \mid x\right) \delta(B \bar{D}) \\
=\left(\begin{array}{c}
B \\
A
\end{array}\right) F\left(\begin{array}{r}
B, A, \vec{C} \\
D, \vec{E}
\end{array} \mid x\right)-\frac{q-1}{q^{2}}\left(\begin{array}{r}
\vec{C} \bar{A} \\
\vec{E} \bar{A}
\end{array}\right) \bar{A}(-x) \delta(D \bar{A}) \\
+\frac{q-1}{q^{2}} A(-1) \bar{D}(x) F\left(\begin{array}{r}
A \bar{D}, \vec{C} \bar{D} \\
\vec{E} \bar{D}
\end{array} \mid x\right) \delta(B),
\end{aligned}
$$

(ii) $\left(\begin{array}{c}C \bar{F} \\ B \bar{F}\end{array}\right) F\left(\begin{array}{r}A, B, C, \vec{D} \\ E, F, \vec{G}\end{array} \mid x\right)-\frac{q-1}{q^{2}}\left(\begin{array}{r}A \bar{B} \\ \bar{B}\end{array}\right)\left(\begin{array}{c}\vec{D} \bar{B} \\ \vec{G} \bar{B}\end{array}\right) B F(-1) \bar{B}(x) \delta(B \bar{E})$

$$
\begin{aligned}
& +\frac{q-1}{q^{2}} B F(-1) F\left(\begin{array}{r}
A, B, \vec{D} \\
E, \vec{G}
\end{array} \mid x\right) \delta(C \bar{F}) \\
= & \left(\begin{array}{r}
C \bar{E} \\
B \bar{E}
\end{array}\right) F\left(\begin{array}{r}
A, C, B, \vec{D} \\
E, F, \vec{G}
\end{array} \mid x\right)-\frac{q-1}{q^{2}}\left(\begin{array}{r}
A \bar{B} \\
\bar{B}
\end{array}\right)\left(\begin{array}{c}
\vec{D} \bar{B} \\
\vec{G} \bar{B}
\end{array}\right) B E(-1) \bar{B}(x) \delta(F \bar{B}) \\
& +\frac{q-1}{q^{2}} B E(-1) F\left(\begin{array}{r}
A, B, \vec{D} \\
F, \vec{G}
\end{array} \mid x\right) \delta(C \bar{E}) .
\end{aligned}
$$

Proof. We use the reduction formulas of Theorem 3.15 as follows. For (i), consider the series

$$
F\left(\begin{array}{r}
A, B, A, \vec{C} \\
A, D, \vec{E}
\end{array} \mid x\right)
$$

This series can be reduced by either Theorem 3.15(iv) or by (v). Setting the two expressions equal results in (i). For (ii), apply Theorem 3.15(v) in two different ways on the series

$$
F\left(\begin{array}{r}
A, B, C, B, \vec{D} \\
E, B, F, \vec{G} \mid x)
\end{array}\right.
$$


COROLlaRY 3.21. If the series is nondegenerate, then

$$
\left(\begin{array}{l}
\vec{B} \\
\vec{C}
\end{array}\right)^{-1} F\left(\begin{array}{r}
A, \vec{B} \\
\vec{C}
\end{array} \mid x\right)
$$

is invariant under any permutation of numerator or denominator parameters.

Proof. Without loss of generality we may restrict our attention to transpositions of numerator parameters. Again as in Theorem 3.20 there are two cases; we will consider the second. By Theorem 3.20(ii), since the series is nondegenerate,

$$
\left(\begin{array}{c}
C \bar{F} \\
B \bar{F}
\end{array}\right) F\left(\begin{array}{c}
A, B, C, \vec{D} \\
E, F, \vec{G}
\end{array} \mid x\right)=\left(\begin{array}{c}
C \bar{E} \\
B \bar{E}
\end{array}\right) F\left(\begin{array}{c}
A, C, B, \vec{D} \\
E, F, \vec{G}
\end{array} \mid x\right) \text {. }
$$

Thus,

$$
\begin{aligned}
& \left\{\left(\begin{array}{l}
B \\
E
\end{array}\right)\left(\begin{array}{l}
C \\
F
\end{array}\right)\left(\begin{array}{l}
\vec{D} \\
\vec{G}
\end{array}\right)\right\}^{-1} F\left(\begin{array}{r}
A, B, C, \vec{D} \\
E, F, \vec{G}
\end{array} \mid x\right) \\
& =\left\{\left(\begin{array}{l}
C \bar{F} \\
B \bar{F}
\end{array}\right)\left(\begin{array}{l}
B \\
E
\end{array}\right)\left(\begin{array}{l}
C \\
F
\end{array}\right)\left(\begin{array}{l}
\vec{D} \\
\vec{G}
\end{array}\right)\right\}^{-1}\left(\begin{array}{l}
C \bar{E} \\
B \bar{E}
\end{array}\right) F\left(\begin{array}{r}
A, C, B, \vec{D} \\
E, F, \vec{G}
\end{array} \mid x\right)
\end{aligned}
$$

and so we must show that

$$
\left(\begin{array}{l}
C \bar{F} \\
B \bar{F}
\end{array}\right)\left(\begin{array}{l}
B \\
E
\end{array}\right)\left(\begin{array}{l}
C \\
F
\end{array}\right)\left(\begin{array}{l}
C \bar{E} \\
B \bar{E}
\end{array}\right)^{-1}=\left(\begin{array}{l}
C \\
E
\end{array}\right)\left(\begin{array}{l}
B \\
F
\end{array}\right)
$$

or

$$
\left(\begin{array}{l}
C \bar{F} \\
B \bar{F}
\end{array}\right)\left(\begin{array}{l}
B \\
E
\end{array}\right)\left(\begin{array}{l}
C \\
F
\end{array}\right)=\left(\begin{array}{l}
C \bar{E} \\
B \bar{E}
\end{array}\right)\left(\begin{array}{l}
C \\
E
\end{array}\right)\left(\begin{array}{l}
B \\
F
\end{array}\right) \text {. }
$$

Since none of the characters involved is trivial, by (2.9) we have

$$
\left(\begin{array}{l}
C \bar{F} \\
B \bar{F}
\end{array}\right)\left(\begin{array}{l}
B \\
E
\end{array}\right)\left(\begin{array}{l}
C \\
F
\end{array}\right)=\frac{G(B) G(C)}{G(E) G(F) G(B \bar{E}) C(B \bar{F}) G(C \bar{B})}=\left(\begin{array}{l}
C \bar{E} \\
B \bar{E}
\end{array}\right)\left(\begin{array}{l}
B \\
F
\end{array}\right)\left(\begin{array}{l}
C \\
E
\end{array}\right) .
$$

Case (i) is similar.

The factor $\left(\frac{\vec{B}}{\vec{C}}\right)$ in Corollary 3.21 arises because we did not normalize hypergeometric series in Definition 3.10. The reason for dropping this factor is that the results in Theorem 3.15 and 3.20 become even worse if this constant is incorporated. It also leads to simpler results in the next section. The complications in Theorems 3.15 and 3.20 might lead one to question the definition we are using for hypergeometric series. The results would be much simpler if one were to use a definition involving Gauss sums instead of Jacobi sums. The justification for our definition comes in the next section when we give transformations and evaluation theorems for hypergeometric functions. These theorems would become intractible using a definition in terms of Gauss sums because the binomial theorem with Gauss sums instead of Jacobi sums is more complicated than Theorem 2.3.

4. Transformations and summation theorems. Classical hypergeometric series satisfy a large number of transformation identities. Kummer gave a list of 24 solutions to the differential equation for the hypergeometric series

$$
x(1-x) y^{\prime \prime}+(c-(a+b+1) x) y^{\prime}-a b y=0 .
$$


Any three such solutions form a linearly dependent set and the resulting linear dependence relations give transformations for hypergeometric series. For an account of these transformations and a list of Kummer's 24 solutions see [18, pp. 283-286 or 4, pp. 105-108].

Kummer's techniques for obtaining solutions for (4.1) involved changes of variables which do not alter the form of the differential equation. We do not have an analogue for (4.1) so we must resort to other means in obtaining transformations. Our techniques are based on the binomial theorem and changes of variables in Definitions 3.5 and 3.10 or in Theorem 3.6.

TheOREM 4.2. For characters $A, B, D, \vec{C}, \vec{E}$ of $\operatorname{GF}(q)$ and $x \in \operatorname{GF}(q)$,

$$
\begin{gathered}
F\left(\begin{array}{r}
A, B, \vec{C} \\
D, \vec{E}
\end{array} \mid x\right)=\bar{D}(x) F\left(\begin{array}{r}
B \bar{D}, A \bar{D}, \vec{C} \bar{D} \\
\bar{D}, \vec{E} \bar{D}
\end{array} \mid x\right), \\
F\left(\begin{array}{r}
A, B, \vec{C} \\
D, \vec{E}
\end{array} \mid x\right)=A B D \vec{C} \vec{E}(-1) \bar{A}(x) F\left(\begin{array}{r}
A, A \bar{D}, A \overrightarrow{\vec{E}} \\
A \bar{B}, A \vec{C}
\end{array} \mid \frac{1}{x}\right) .
\end{gathered}
$$

Proof. We change variables in Definition 3.10,

$$
F\left(\begin{array}{r}
A, B, \vec{C} \\
D, \vec{E}
\end{array} \mid \chi\right)=\frac{q}{q-1} \sum_{\chi}\left(\begin{array}{c}
A \chi \\
\chi
\end{array}\right)\left(\begin{array}{c}
B \chi \\
D \chi
\end{array}\right)\left(\begin{array}{c}
\vec{C} \chi \\
\vec{E} \chi
\end{array}\right) \chi(x) .
$$

For (i), replace $\chi$ by $\bar{D} \chi$, for (ii), replace $\chi$ by $\overline{A \chi}$ and appeal to property (2.8) for binomial coefficients.

We remark that the change of variables in (i) can be carried out for any denominator parameter, and the change of variables in (ii) can be done for any numerator parameter to obtain similar transformations. The result in (ii) is the analogue for the classical result on reversing the summation of a terminating hypergeometric series [17, p. 48]

$$
\begin{aligned}
{ }_{n+1} & F_{n}\left(\begin{array}{r}
-m, a_{1}, \ldots, a_{n} \\
b_{1}, \ldots, b_{n}
\end{array} \mid x\right) \\
& =\frac{\left(a_{1}\right)_{m} \cdots\left(a_{n}\right)_{m}}{\left(b_{1}\right)_{m} \cdots\left(b_{n}\right)_{m}}(-x)^{m}{ }_{n+1} F_{n}\left(\begin{array}{r}
-m, 1-b_{1}-m, \ldots, 1-b_{n}-m \\
1-a_{1}-m, \ldots, 1-a_{n}-m
\end{array} \mid \frac{1}{x}\right) .
\end{aligned}
$$

It is interesting to note that because the character sum has only a finite number of terms, the analogy is strongest with terminating hypergeometric series. In fact, if there is a difference between terminating and nonterminating cases, our analogue will be with the terminating case of a formula. This phenomenon can be seen in the first part of the following theorem.

TheOREM 4.4. For characters $A, B, C$ of $\mathrm{GF}(q)$ and $x \in \mathrm{GF}(q)$,

$$
\begin{aligned}
{ }_{2} F_{1}\left(\begin{array}{r}
A, B \\
C
\end{array} \mid x\right)= & A(-1)_{2} F_{1}\left(\begin{array}{c}
A, B \\
A B \bar{C}
\end{array} \mid 1-x\right) \\
& +A(-1)\left(\begin{array}{c}
B \\
\bar{A} C
\end{array}\right) \delta(1-x)-\left(\begin{array}{l}
B \\
C
\end{array}\right) \delta(x),
\end{aligned}
$$


(ii)

$$
\begin{aligned}
{ }_{2} F_{1}\left(\begin{array}{r}
A, B \\
C
\end{array} \mid x\right)= & C(-1) \bar{A}(1-x){ }_{2} F_{1}\left(\begin{array}{r}
A, C \bar{B} \\
C
\end{array} \mid \frac{x}{x-1}\right) \\
& +A(-1)\left(\frac{B}{A C}\right) \delta(1-x),
\end{aligned}
$$

(iii)

$$
\begin{aligned}
{ }_{2} F_{1}\left(\begin{array}{r}
A, B \\
C
\end{array} \mid x\right)= & \bar{B}(1-x){ }_{2} F_{1}\left(\begin{array}{r}
C \bar{A}, B \\
C
\end{array} \mid \frac{x}{x-1}\right) \\
& +A(-1)\left(\frac{B}{\bar{A} C}\right) \delta(1-x),
\end{aligned}
$$

(iv)

$$
\begin{aligned}
{ }_{2} F_{1}\left(\begin{array}{r}
A, B \\
C
\end{array} \mid x\right)= & C(-1) C \overline{A B}(1-x){ }_{2} F_{1}\left(\begin{array}{r}
C \bar{A}, C \bar{B} \\
C
\end{array} \mid x\right) \\
& +A(-1)\left(\begin{array}{c}
B \\
\bar{A} C
\end{array}\right) \delta(1-x) .
\end{aligned}
$$

PROOF. We change variables in the definition

$$
{ }_{2} F_{1}\left(\begin{array}{r}
A, B \\
C
\end{array} \mid x\right)=\varepsilon(x) \frac{B C(-1)}{q} \sum_{y} B(y) \bar{B} C(1-y) \bar{A}(1-x y) .
$$

For (i), we set $y=z /(z-1)$. Then we have $1-y=1 /(1-z)$ and $1-x y=$ $(1-(1-x) z) /(1-z)$. Thus,

$$
\begin{aligned}
{ }_{2} F_{1}\left(\begin{array}{rl}
A & B \\
& C
\end{array} \mid x\right) \\
=\varepsilon(x) \frac{B C(-1)}{q} \sum_{z} B(z) \bar{B}(z-1) B \bar{C}(1-z) \bar{A}(1-z) \bar{A}(1-(1-x) z) \\
=\varepsilon(x) A(-1) \frac{A C(-1)}{q} \sum_{z} B(z) A \bar{C}(1-z) \bar{A}(1-(-x) z) .
\end{aligned}
$$

The result now follows from the observation that $\varepsilon(x)=\varepsilon(1-x)+\delta(1-x)-$ $\delta(x)$. For (ii), (iii) and (iv) make the changes of variables

$$
y=1-z, \quad y=\frac{z}{1-x+x z} \quad \text { and } \quad y=\frac{1-z}{1-x z}, \quad \text { respectively. }
$$

Theorem 4.4, parts (ii) and (iii) are analogues for Pfaff's transformation [17, p. 31]

$$
{ }_{2} F_{1}\left({ }^{a}, b \mid x\right)=(1-x)^{-a}{ }_{2} F_{1}\left(a, c-b\left|\frac{x}{c}\right| \frac{x-1}{x-1}\right),
$$

part (iv) is an analogue for Euler's transformation [17, p. 10]

$$
{ }_{2} F_{1}(a, b \mid x)=(1-x)^{c-a-b}{ }_{2} F_{1}(c-a, c-b|x| x) .
$$

Part (i) is an analogue for the transformation [17, p. 34]

$$
\begin{aligned}
{ }_{2} F_{1}\left(\begin{array}{c}
a, \\
{ }_{1+a}
\end{array}\right. & +b-c \mid 1-x)=\frac{\Gamma(1+a+b-c) \Gamma(1-c)}{\Gamma(1+a-c) \Gamma(1+b-c)}{ }_{2} F_{1}(a, b \mid x) \\
& +\frac{\Gamma(1+a+b-c) \Gamma(c-1)}{\Gamma(a) \Gamma(b)} x^{1-c}{ }_{2} F_{1}\left(\begin{array}{c}
1+a-c, 1+b-c \\
2-c
\end{array} \mid x\right) .
\end{aligned}
$$


To strengthen the analogy, note that if $a$ is a negative integer, say $a=-n$, then $1 / \Gamma(a)=0$ so (4.7) becomes, in the terminating version,

$$
{ }_{2} F_{1}\left(\begin{array}{r}
-n, b \\
c
\end{array} \mid x\right)=\frac{(c-b)_{n}}{(c)_{n}} F_{2}\left(\begin{array}{cc}
-n, & b \\
& 1-n+b-c
\end{array} \mid 1-x\right),
$$

which agrees well with 4.4(i).

It may be pointed out that if one includes the trivial $y \mapsto z$ then there are 24 changes of variables of the type used in the proof of Theorem 4.4. This gives rise to 23 transformations ( 24 if one includes the identity transformation). These 24 changes of variables and the resulting transformations correspond to Kummer's 24 solutions. We have listed the most important transformations. The others may be obtained by various compositions of the transformations given in Theorem 4.2, in the case of the ${ }_{2} F_{1}$, with the transformations in Theorem 4.4.

TheOREM 4.9. For characters $A, B$ and $C$ of $\operatorname{GF}(q)$,

$$
{ }_{2} F_{1}\left(\begin{array}{r|}
A, B \\
C
\end{array} \mid 1\right)=A(-1)\left(\begin{array}{r}
B \\
\overline{A C}
\end{array}\right) \text {. }
$$

Proof. Set $x=1$ in Theorem 4.4. Alternatively, one could set $x=1$ in the definition of $\mathrm{a}_{2} F_{1}$ :

$$
\begin{aligned}
{ }_{2} F_{1}\left(\begin{array}{r}
A, B \\
C
\end{array} \mid 1\right) & =\frac{B C(-1)}{q} \sum_{y} B(y) \bar{B} C(1-y) \bar{A}(1-y) \\
& =\frac{B C(-1)}{q} J(B, \overline{A B} C)=A(-1)\left(\begin{array}{r}
B \\
\overline{A C}
\end{array}\right) .
\end{aligned}
$$

Theorem 4.9 is the analogue of Gauss's evaluation [2, p. 2]

$$
{ }_{2} F_{1}\left(\begin{array}{r}
a, b \\
c
\end{array} \mid\right)=\frac{\Gamma(c) \Gamma(c-a-b)}{\Gamma(c-a) \Gamma(c-b)} .
$$

In fact, if $F$ is nondegenerate, then putting in the proper normalization yields

$$
\begin{aligned}
\left(\begin{array}{l}
B \\
C
\end{array}\right)^{-1}{ }_{2} F_{1}\left(\begin{array}{r}
A, B \\
C
\end{array} \mid 1\right) & =A(-1)\left(\begin{array}{c}
B \\
\bar{A} C
\end{array}\right)\left(\begin{array}{l}
B \\
C
\end{array}\right)^{-1} \\
& =A(-1) \frac{G(C) G(B \bar{C})}{G(C \bar{A}) G(A B \bar{C})}=\frac{G(C) G(C \overline{A B})}{G(C \bar{A}) G(C \bar{B})} .
\end{aligned}
$$

Definition 3.5 leads to other summation theorems as well. For example, if we set $x=-1$ we have

$$
{ }_{2} F_{1}\left(\begin{array}{r}
A, B \\
C
\end{array} \mid-1\right)=\frac{B C(-1)}{q} \sum_{y} B(y) \bar{B} C(1-y) \bar{A}(1+y) .
$$


If $C=\overline{A B}$ this becomes

$$
\begin{aligned}
{ }_{2} F_{1}\left(\begin{array}{r}
A, B \\
\overline{A B} B
\end{array} \mid-1\right) & =\frac{A(-1)}{q} \sum_{y} B(y) \bar{A}\left(1-y^{2}\right) \\
& =\frac{A(-1)}{q} \sum_{y} B(y) \frac{q}{q-1} \sum_{\chi}\left(\begin{array}{r}
A \chi \\
\chi
\end{array}\right) \chi\left(y^{2}\right) \\
& =\frac{A(-1)}{q-1} \sum_{\chi}\left(\begin{array}{r}
A \chi \\
\chi
\end{array}\right) \sum_{y} B \chi^{2}(y) .
\end{aligned}
$$

The inner sum is zero unless $B \chi^{2}$ is trivial so we are lead to

$$
{ }_{2} F_{1}\left(\begin{array}{r}
A, B \\
\bar{A} B
\end{array} \mid-1\right)= \begin{cases}0 & \text { if } B \text { is not a } \\
\left(\begin{array}{l}
C \\
A
\end{array}\right)+\left(\begin{array}{r}
\varphi C \\
A
\end{array}\right) & \text { if } B=C^{2} .\end{cases}
$$

This result is the analogue of Kummer's theorem [2, p. 9]

$$
{ }_{2} F_{1}\left(\begin{array}{c}
a, \quad b \\
1+b-a
\end{array} \mid-1\right)=\frac{\Gamma(1+b-a) \Gamma\left(1+\frac{1}{2} b\right)}{\Gamma(1+b) \Gamma\left(1+\frac{1}{2} b-a\right)} .
$$

The terminating form of $(4.12)$ is

$$
{ }_{2} F_{1}\left(\begin{array}{ll}
a, \quad-n \\
1-a-n
\end{array} \mid-1\right)= \begin{cases}0 & \text { if } n \text { is odd, } \\
\frac{(N)_{N}}{(a+N)_{N}} & \text { if } n=2 N .\end{cases}
$$

Using Theorem 4.4 we are lead to

$$
\begin{aligned}
& (A, B) \quad \text { if } B \text { is not a square, } \\
& { }_{2} F_{1}\left(\begin{array}{r}
A, B \\
A^{2}
\end{array} \mid 2\right)=A(-1)\left\{\left(\begin{array}{l}
C \\
A
\end{array}\right)+\left(\begin{array}{c}
\varphi C \\
A
\end{array}\right) \quad \text { if } B=C^{2}\right. \\
& { }_{2} F_{1}\left(\begin{array}{rl}
A, \bar{A} & \frac{1}{2} \\
\overline{A B}
\end{array}\right)=A(-2) \begin{cases}0 & \text { if } B \text { is not a } \\
\left(\begin{array}{l}
C \\
A
\end{array}\right)+\left(\begin{array}{r}
\varphi C \\
A
\end{array}\right) & \text { if } B=C^{2} .\end{cases}
\end{aligned}
$$

A large number of quadratic transformations exist for classical hypergeometric series. A partial list of quadratic transformations can be found in [4, pp. 64-67], which also gives necessary and sufficient conditions for the existence of a quadratic transformation in terms of the parameters of the function. A similar result holds for hypergeometric series over $\mathrm{GF}(q)$, but it is not our intention to examine the problem in such depth. Instead we will derive a single quadratic transformation by way of example.

TheOREM 4.16. For characters $A$ and $B$ of $\mathrm{GF}(q)$ and $x \in \mathrm{GF}(q)$,

$$
\begin{aligned}
& { }_{2} F_{1}\left(\begin{array}{r}
A, B \\
\overline{A B}
\end{array} \mid x\right)=\delta(1+x)\left(\begin{array}{l}
0 \\
\left(\begin{array}{c}
C \\
A
\end{array}\right)+\left(\begin{array}{r}
\varphi C \\
A
\end{array}\right) \quad \text { if } B=C^{2}
\end{array}\right. \\
& \quad+\frac{A(-1)}{q} B\left(\frac{1+x}{x}\right)+\bar{B}(1+x) \frac{q}{q-1} \sum_{\chi}\left(\begin{array}{c}
B \chi^{2} \\
\chi
\end{array}\right)\left(\begin{array}{c}
B \chi \\
\overline{A B} \chi
\end{array}\right) \chi\left(\frac{x}{(1+x)^{2}}\right) .
\end{aligned}
$$


Proof. Using the definition for $\mathrm{a}_{2} F_{1}$ we have

$$
\begin{aligned}
{ }_{2} F_{1}\left(\begin{array}{r}
A, B \\
\overline{A B}
\end{array} \mid x\right) & =\varepsilon(x) \frac{A(-1)}{q} \sum_{y} B(y) \bar{A}(1-y) \bar{A}(1-x y) \\
& =\varepsilon(x) \frac{A(-1)}{q} \sum_{y} B(y) \bar{A}(1-y(1+x-x y)) .
\end{aligned}
$$

By the binomial theorem,

$$
\begin{aligned}
\bar{A}(1-y(1+x-x y)) & \\
& =\delta(y(1+x-x y))+\frac{q}{q-1} \sum_{\chi}\left(\begin{array}{r}
A \chi \\
\chi
\end{array}\right) \chi(y) \chi(1+x-x y) .
\end{aligned}
$$

Using this in (4.16) and changing $y$ to $y / x$ gives

$$
\begin{aligned}
{ }_{2} F_{1}\left(\begin{array}{r}
A, B \\
\overline{A B}
\end{array} \mid x\right)= & \frac{A(-1)}{q} B\left(\frac{1+x}{x}\right)+\frac{A(-1)}{q-1} \\
& \cdot \sum_{\chi, y}\left(\begin{array}{r}
A \chi \\
\chi
\end{array}\right) \overline{B \chi}(x) B \chi(y) \chi(1+x-y) .
\end{aligned}
$$

Since $1=\varepsilon(1+x)+\delta(1+x),(4.17)$ becomes

$$
\begin{aligned}
{ }_{2} F_{1}\left(\begin{array}{r}
A, B \\
\bar{A} B
\end{array} \mid x\right)= & \frac{A(-1)}{q} B\left(\frac{1+x}{x}\right)+\delta(1+x) \frac{A(-1)}{q-1} \sum_{\chi, y}\left(\begin{array}{r}
A \chi \\
\chi
\end{array}\right) B(-1) B \chi^{2}(y) \\
& +\varepsilon(1+x) \frac{A(-1)}{q-1} \sum_{\chi, y}\left(\begin{array}{r}
A \chi \\
\chi
\end{array}\right) \overline{B \chi}(x) B \chi(y) \chi(1+x-y) .
\end{aligned}
$$

The second term on the right-hand side was evaluated in obtaining (4.11). To evaluate the third term make the change of variables $y \mapsto(1+x) y$ to obtain

$$
\begin{aligned}
& { }_{2} F_{1}\left(\begin{array}{r}
A, B \\
\overline{A B}
\end{array} \mid x\right)=\frac{A(-1)}{q} B\left(\frac{1+x}{x}\right)+\delta(1+x)\left\{\begin{array}{l}
0 \text { if } B \text { is not a square, } \\
\left(\begin{array}{c}
C \\
A
\end{array}\right)+\left(\begin{array}{r}
\varphi C \\
A
\end{array}\right) \text { if } B=C^{2},
\end{array}\right. \\
& +B\left(\frac{1+x}{x}\right) \frac{A(-1)}{q-1} \sum_{\chi}\left(\begin{array}{r}
A \chi \\
\chi
\end{array}\right) \chi\left(\frac{(1+x)^{2}}{x}\right) \sum_{y} B \chi(y) \chi(1-y) .
\end{aligned}
$$

The inner sum on this last term is

$$
J(B \chi, \chi)=q \chi(-1)\left(\begin{array}{r}
B \chi \\
\bar{\chi}
\end{array}\right) .
$$

Using this and making the change $\chi \mapsto \overline{B \chi}$ gives the desired result.

Theorem 4.16 gives the best analogue available for the classical transformation [ 4 , p. 113]

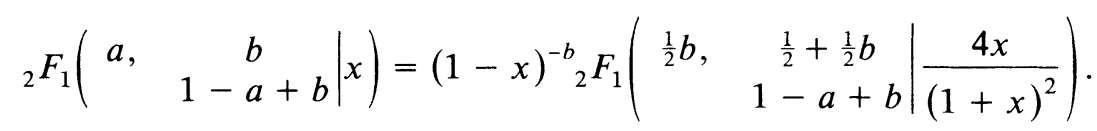


It is somewhat disappointing, however, that the major term on the right-hand side of (4.16) does not, strictly speaking, represent a hypergeometric series. The problem lies in the term $\left(\begin{array}{c}B \chi^{2} \\ \chi\end{array}\right)$. If $B$ is a square then we can exploit (2.16) to improve the analogy.

THEOREM 4.20. For characters $A$ and $B$ of $\mathrm{GF}(q)$ and $x \in \operatorname{GF}(q)$,

$$
\begin{aligned}
{ }_{2} F_{1}\left(\begin{array}{c}
A, B^{2} \\
\bar{A} B^{2}
\end{array}\right)= & \delta(1+x)\left[\left(\begin{array}{c}
B \\
A
\end{array}\right)+\left(\begin{array}{r}
\varphi B \\
A
\end{array}\right)\right] \\
& +A(-1) \frac{q-1}{q} \varepsilon(x) \varepsilon(1+x) \delta(\varphi B) \\
& +\frac{q-1}{q^{2}}\left(\begin{array}{r}
\varphi \\
\varphi B
\end{array}\right)^{-1} A(-1) B^{2}\left(\frac{2}{1-x}\right) \varepsilon(x) \varepsilon(1+x) \delta(A \bar{B}) \\
& +\left(\begin{array}{c}
B \\
A
\end{array}\right)\left(\begin{array}{r}
\varphi \\
\varphi B
\end{array}\right)^{-1} A(-1) B^{2}\left(\frac{2}{1+x}\right){ }_{2} F_{1}\left(\begin{array}{c}
\varphi B, B \\
\bar{A} B^{2}
\end{array} \mid \frac{4 x}{(1+x)^{2}}\right) .
\end{aligned}
$$

Proof. It follows from (2.16) that

$$
\left(\begin{array}{c}
B^{2} \chi^{2} \\
\chi
\end{array}\right)=\left(\begin{array}{c}
\varphi B \chi \\
\chi
\end{array}\right)\left(\begin{array}{c}
B \chi \\
B^{2} \chi
\end{array}\right)\left(\begin{array}{r}
\varphi \\
\varphi B
\end{array}\right)^{-1} B \chi(4) .
$$

Consequently, if $B$ is replaced by $B^{2}$ in Theorem 4.16 we obtain

$$
\begin{aligned}
{ }_{2} F_{1}\left(\begin{array}{r}
A, B^{2} \\
\bar{A} B^{2}
\end{array} \mid x\right) & =\delta(1+x)\left[\left(\begin{array}{c}
B \\
A
\end{array}\right)+\left(\begin{array}{r}
\varphi B \\
A
\end{array}\right)\right]+\frac{A(-1)}{q} B^{2}\left(\frac{1+x}{x}\right) \\
& +\bar{B}^{2}(1+x) B(4)\left(\begin{array}{r}
\varphi \\
\varphi B
\end{array}\right)^{-1} \frac{q}{q-1} \sum_{\chi}\left(\begin{array}{c}
\varphi B \chi \\
\chi
\end{array}\right)\left(\begin{array}{c}
B \chi \\
B^{2} \chi
\end{array}\right)\left(\begin{array}{c}
B^{2} \chi \\
\bar{A} B^{2} \chi
\end{array}\right) \chi\left(\frac{4 x}{(1+x)^{2}}\right) .
\end{aligned}
$$

The character sum on the right-hand side is a degenerate ${ }_{3} F_{2}$ which when reduced to $\mathrm{a}_{2} F_{1}$ gives rise to the desired result.

Given the quadratic transformation in Theorem 4.20, others may now be obtained by using the ${ }_{2} F_{1}$-linear transformations. More information on quadratic transformations over finite fields can be found in [9, pp. 40-55].

Transformations for higher order hypergeometric series also exist. In fact, ${ }_{3} F_{2}(1)$ transformations follow from ${ }_{2} F_{1}(x)$ transformations. For example, if both sides of the result in Theorem 4.4(i) are multiplied by $(D E(-1) / q) D(x) \bar{D} E(1-x)$, then summing on $x$ gives

$$
\begin{aligned}
& \frac{D E(-1)}{q} \sum_{x}{ }_{2} F_{1}\left(\begin{array}{r}
A, B \\
C
\end{array} \mid x\right) D(x) \bar{D} E(1-x) \\
& \quad=\frac{A D E(-1)}{q} \sum_{x}{ }_{2} F_{1}\left(\begin{array}{c}
A, B \\
A B \bar{C}
\end{array} \mid 1-x\right) D(x) \bar{D} E(1-x) .
\end{aligned}
$$

If we replace $x$ by $1-x$ on the right-hand side, then by Theorem 3.13 we have

$$
{ }_{3} F_{2}\left(\begin{array}{r}
A, B, D \\
C, E
\end{array} \mid 1\right)=A E(-1)_{3} F_{2}\left(\begin{array}{ccc}
A, & B, & \bar{D} E \\
& A B \bar{C}, & E
\end{array} \mid 1\right) .
$$


Similarly, from Theorem 4.4, parts (ii), (iii) and (iv) we obtain

$$
\begin{gathered}
{ }_{3} F_{2}\left(\begin{array}{r}
A, B, D \\
C, E
\end{array}\right)=A C(-1)_{3} F_{2}\left(\begin{array}{rcc}
A, & C \bar{B}, & D \\
C, & A D \bar{E}
\end{array}\right), \\
{ }_{3} F_{2}\left(\begin{array}{r}
A, B, D \mid 1 \\
C, E
\end{array}\right)=B(-1)_{3} F_{2}\left(\begin{array}{ccc}
C \bar{A}, & B, & D \\
& C, & B D \bar{E}
\end{array}\right),
\end{gathered}
$$

and

$$
{ }_{3} F_{2}\left(\begin{array}{r}
A, B, D \\
C, E
\end{array} \mid 1\right)=A B(-1)_{3} F_{2}\left(\begin{array}{ccc}
C \bar{A}, & C \bar{B}, & D \\
& C, & \overline{A B} C E
\end{array} \mid 1\right),
$$

respectively.

There exists $\mathrm{a}_{3} F_{2}$ quadratic transformation [17, p. 90],

$$
\begin{aligned}
& { }_{3} F_{2}\left(\begin{array}{l}
a, b, \quad c \\
1+c-a, 1+c-b
\end{array} \mid x\right) \\
& =(1-x)_{3}^{-c} F_{2}\left(\begin{array}{cc|c}
\frac{1}{2} c, & \frac{1}{2}+\frac{1}{2} c, & 1+c-a-b \\
1+c-a, & 1+c-b & \frac{-4 x}{(1-x)^{2}}
\end{array}\right) .
\end{aligned}
$$

As might be expected, a similar result holds for the finite field case.

TheOREM 4.28. For characters $A, B$ and $C$ of $\mathrm{GF}(q)$ and $x \in \mathrm{GF}(q)$,

$$
\begin{aligned}
& { }_{3} F_{2}\left(\begin{array}{ccc}
A, & B, & C \\
& C \bar{A}, & C \bar{B}
\end{array} \mid x\right)=-\frac{q-1}{q^{2}} A C(-1) \varepsilon(x) \delta(b) \\
& +\frac{q-1}{q^{2}} B C(-1) \varepsilon(x) \varepsilon(1+x) \delta(A)-\frac{q-1}{q^{2}} \bar{A}(-x) \delta(\overline{A B} C) \\
& +\frac{1}{q} A B C(-1) \delta(1+x)+\frac{1}{q}\left(\begin{array}{r}
B \\
A B
\end{array}\right) B C(-1) C\left(\frac{x-1}{x}\right) \\
& +\delta(1-x) A B(-1)\left\{\begin{array}{l}
0 \quad \text { if } C \text { is not a square } \\
\left(\begin{array}{l}
D \\
A
\end{array}\right)\left(\begin{array}{r}
B \bar{D} \\
A B \bar{D}
\end{array}\right)+\left(\begin{array}{r}
\varphi D \\
A
\end{array}\right)\left(\begin{array}{r}
\varphi B \bar{D} \\
\varphi A B \bar{D}
\end{array}\right) \quad \text { if } C=D^{2}
\end{array}\right. \\
& +B C(-1) \bar{C}(1-x) \frac{q}{q-1} \sum_{\chi}\left(\begin{array}{c}
C \chi^{2} \\
\chi
\end{array}\right)\left(\begin{array}{c}
C \chi \\
\bar{A} C \chi
\end{array}\right)\left(\begin{array}{r}
\overline{A B} C \chi \\
\bar{B} C \chi
\end{array}\right) \chi\left(\frac{-x}{(1-x)^{2}}\right) \text {. }
\end{aligned}
$$

SKETCH OF PROOF. We proceed similarly to the proof of Theorem 4.16, beginning with the analogue of the integral representation, Corollary 3.14(i),

$$
\text { (4.29) } \begin{aligned}
{ }_{3} F_{2}\left(\begin{array}{ccc}
A, & B, & C \\
C \bar{A}, & C \bar{B} \mid x
\end{array}\right) \\
\quad=\varepsilon(x) \frac{A C(-1)}{q^{2}} \sum_{y, z} C(y) \bar{B}(1-y) B(z) \overline{A B} C(1-z) \bar{A}(1-x y z) .
\end{aligned}
$$


Expanding $\bar{A}(1-z) \bar{A}(1-x y z)=\bar{A}(1-z(1+x y-x y z))$ by the binomial theorem and taking $z$ to $1-z$ gives

$$
\begin{aligned}
& { }_{3} F_{2}\left(\begin{array}{ccc}
A, \quad B, & C \\
C \bar{A}, & C \bar{B}
\end{array} \mid x\right)=\varepsilon(x) \frac{A C(-1)}{q^{2}} \frac{q}{q-1} \sum_{\chi}\left(\begin{array}{c}
A \chi \\
\chi
\end{array}\right) \sum_{y, z} C(y) \\
& \cdot \bar{B}(1-y) \bar{B} C(z) B \chi(1-z) \chi(1+x y z) \\
& =A C(-1) \frac{q}{q-1} \sum_{\chi}\left(\begin{array}{r}
A \chi \\
\chi
\end{array}\right) \chi(-1)_{3} F_{2}\left(\begin{array}{ccc}
\bar{\chi}, & \bar{B} C, & C \\
& C \chi, & \bar{B} C
\end{array} \mid-\chi\right),
\end{aligned}
$$

again by Corollary 3.14(i). When the ${ }_{3} F_{2}$ on the right-hand side is reduced, the main term is

$$
A B C(-1) \frac{q}{q-1} \sum_{\chi}\left(\begin{array}{r}
A \chi \\
\chi
\end{array}\right)\left(\begin{array}{r}
B \chi \\
\chi
\end{array}\right) \chi(-1)_{2} F_{1}\left(\begin{array}{cc}
\bar{\chi}, & C \\
& C \chi
\end{array} \mid-\chi\right) .
$$

One may now apply the ${ }_{2} F_{1}$-quadratic transformation producing the main term

$$
\begin{aligned}
A B C( & -1) \frac{q}{q-1} \sum_{\chi}\left(\begin{array}{r}
A \chi \\
\chi
\end{array}\right)\left(\begin{array}{r}
B \chi \\
\chi
\end{array}\right) \chi(-1) \bar{C}(1-x) \frac{q}{q-1} \\
& \cdot \sum_{\psi}\left(\begin{array}{c}
C \psi^{2} \\
\psi
\end{array}\right)\left(\begin{array}{r}
C \psi \\
C \chi \psi
\end{array}\right) \psi\left(\frac{-x}{(1-x)^{2}}\right) \\
= & A B(-1) \bar{C}(1-x) \frac{q}{q-1} \sum_{\psi}\left(\begin{array}{c}
C \psi^{2} \\
\psi
\end{array}\right) \psi\left(\frac{x}{(1-x)^{2}}\right){ }_{3} F_{2}\left(\begin{array}{ccc}
A, & B, & \varepsilon \\
\varepsilon, & C \psi
\end{array}\right) .
\end{aligned}
$$

The above ${ }_{3} F_{2}$ reduces to $\mathrm{a}_{2} F_{1}(1)$ which can be evaluated by Theorem 4.9 to obtain the summation on the right-hand side of Theorem 4.28.

As was the case with Theorem 4.16, more can be said if the appropriate character is a square. For simplicity we will only state the result in the nondegenerate case.

Corollary 4.30. If $A, B, \varphi C, C \bar{A}$ and $C^{2} \overline{A B}$ are all nontrivial, then

$$
\begin{aligned}
{ }_{3} F_{2}\left(\begin{array}{rrr}
A, & B, & C^{2} \\
C^{2} \bar{A}, & C^{2} \bar{B}
\end{array} \mid x\right) \\
=\delta(1-x) A B(-1)\left[\left(\begin{array}{l}
C \\
A
\end{array}\right)\left(\begin{array}{r}
\bar{C} B \\
A B \bar{C}
\end{array}\right)+\left(\begin{array}{r}
\varphi C \\
A
\end{array}\right)\left(\begin{array}{r}
\varphi \bar{C} B \\
\varphi A B \bar{C}
\end{array}\right)\right]+\frac{A B(-1)}{q} \delta(1+x) \\
+\left(\begin{array}{c}
C \\
A
\end{array}\right)\left(\begin{array}{r}
\varphi \\
\varphi C
\end{array}\right)^{-1} A B(-1) C^{2}\left(\frac{2}{1-x}\right){ }_{3} F_{2}\left(\begin{array}{ccc}
\varphi, & C, & C^{2} \overline{A B} \\
C^{2} \bar{A}, & C^{2} \bar{B} & \frac{-4 x}{(1-x)^{2}}
\end{array} .\right.
\end{aligned}
$$

Proof. Similar to that of Theorem 4.20.

We close this section with some important ${ }_{3} F_{2}(1)$ evaluations. In the classical case there are four major summation theorems. These are Saalschütz's theorem [17, p. 49]

$$
{ }_{3} F_{2}\left(\begin{array}{c}
-n, a, \quad b \\
c, 1-c+a+b-n
\end{array} \mid 1\right)=\frac{(c-a)_{n}(c-b)_{n}}{(c)_{n}(c-a-b)_{n}},
$$


Dixon's theorem [17, p. 51]

$$
\begin{aligned}
& \text { (4.32) }{ }_{3} F_{2}\left(\begin{array}{cc}
a, & b, \quad c \\
& 1+a-b, 1+a-c
\end{array} \mid\right. \\
& =\frac{\Gamma\left(1+\frac{1}{2} a\right) \Gamma(1+a-b) \Gamma(1+a-c) \Gamma\left(1+\frac{1}{2} a-b-c\right)}{\Gamma(1+a) \Gamma\left(1+\frac{1}{2} a-b\right) \Gamma\left(1+\frac{1}{2} a-c\right) \Gamma(1+a-b-c)},
\end{aligned}
$$

Watson's theorem $[17$, p. 54]

$$
\begin{aligned}
& { }_{3} F_{2}\left(\begin{array}{cc|c}
a, & b, & c \\
& \frac{1}{2}(1+a+b), 2 c
\end{array} \mid 1\right) \\
& =\frac{\Gamma\left(\frac{1}{2}\right) \Gamma\left(\frac{1}{2}+c\right) \Gamma\left(\frac{1}{2}(1+a+b)\right) \Gamma\left(\frac{1}{2}(1-a-b)+c\right)}{\Gamma\left(\frac{1}{2}(1+a)\right) \Gamma\left(\frac{1}{2}(1+b)\right) \Gamma\left(\frac{1}{2}(1-a)+c\right) \Gamma\left(\frac{1}{2}(1-b)+c\right)},
\end{aligned}
$$

and Whipple's theorem [16, p. 54]

$$
\begin{aligned}
& \text { (4.34) }{ }_{3} F_{2}\left(\begin{array}{cc}
a, & 1-a, \\
b, & 1-b+2 c
\end{array} \mid\right) \\
& =\frac{\pi \Gamma(b) \Gamma(1-b+2 c)}{2^{2 c-1} \Gamma\left(\frac{1}{2}(a+b)\right) \Gamma\left(\frac{1}{2}(1+a-b+2 c)\right) \Gamma\left(\frac{1}{2}(1-a+b)\right) \Gamma\left(\frac{1}{2}(2-a-b+2 c)\right)} .
\end{aligned}
$$

We give analogues for each of these.

Theorem 4.35. For characters $A, B, C$ and $D$ of $\operatorname{GF}(q)$,

$$
{ }_{3} F_{2}\left(\begin{array}{rc}
A, B, & C \\
D, & A B C \bar{D}
\end{array} \mid 1\right)=B C(-1)\left(\begin{array}{r}
C \\
D \bar{A}
\end{array}\right)\left(\begin{array}{r}
B \\
D \bar{C}
\end{array}\right)-\frac{1}{q} B D(-1)\left(\begin{array}{r}
D \bar{B} \\
A
\end{array}\right) .
$$

Proof. Recall Theorem 4.4(iv),

$$
\begin{aligned}
{ }_{2} F_{1}\left(\begin{array}{r}
A, B \\
C \mid x
\end{array}\right) & C(-1) C \overline{A B}(1-x)_{2} F_{1}\left(\begin{array}{r}
C \bar{A}, C \bar{B} \\
C
\end{array} \mid x\right) \\
& +A(-1)\left(\begin{array}{r}
B \\
\bar{A} C
\end{array}\right) \delta(1-x) .
\end{aligned}
$$

We calculate the coefficient of $D(x)$ on both sides of (4.36). The coefficient of $D(x)$ in

is

$$
{ }_{2} F_{1}\left(\begin{array}{r|r}
A, B \\
C
\end{array}\right)
$$

Since

$$
\frac{q}{q-1}\left(\begin{array}{r}
A D \\
D
\end{array}\right)\left(\begin{array}{l}
B D \\
C D
\end{array}\right)
$$

$$
\delta(1-x)=\frac{1}{q-1} \sum_{\chi} \chi(x)
$$

the coefficient of $D(x)$ in $A(-1)\left(\frac{B}{A C}\right) \delta(1-x)$ is

$$
\frac{1}{q-1} A(-1)\left(\begin{array}{r}
B \\
\bar{A} C
\end{array}\right) \text {. }
$$

By the binomial theorem, the coefficient of $D(x)$ in $\chi(x) C \overline{A B}(1-x)$ is

$$
\frac{q}{q-1}\left(\begin{array}{c}
C \overline{A B} \\
D \bar{\chi}
\end{array}\right) D \chi(-1) \text {. }
$$


Collecting terms, we have

$$
\begin{aligned}
\frac{q}{q-1}\left(\begin{array}{r}
A D \\
D
\end{array}\right)\left(\begin{array}{l}
B D \\
C D
\end{array}\right)= & D(-1)\left(\frac{q}{q-1}\right)^{2} \sum_{\chi}\left(\begin{array}{c}
C \overline{A \chi} \\
\chi
\end{array}\right)\left(\begin{array}{c}
C \bar{B} \chi \\
C \chi
\end{array}\right)\left(\begin{array}{r}
C \overline{A B} \\
D \bar{\chi}
\end{array}\right) \chi(-1) \\
& +\frac{1}{q-1} A(-1)\left(\begin{array}{c}
B \\
\overline{A C}
\end{array}\right)
\end{aligned}
$$

or

$$
\left(\begin{array}{c}
A D \\
D
\end{array}\right)\left(\begin{array}{c}
B D \\
C D
\end{array}\right)-\frac{1}{q} A(-1)\left(\begin{array}{c}
B \\
\bar{A} C
\end{array}\right)=A B C(-1) \frac{q}{q-1} \sum_{\chi}\left(\begin{array}{c}
C \bar{A} \chi \\
\chi
\end{array}\right)\left(\begin{array}{c}
C \bar{B} \chi \\
C \chi
\end{array}\right)\left(\begin{array}{c}
\bar{D} \chi \\
C \overline{A B D} \chi
\end{array}\right) .
$$

The result now follows by relabeling the parameters.

Theorem 4.37. For characters $A, B$ and $C$ of $\mathrm{GF}(q)$,

$$
\begin{aligned}
{ }_{3} F_{2}\left(\begin{array}{cc}
A, \quad B, \quad C \\
C \bar{A}, \quad C \bar{B}
\end{array} \mid\right)= & \frac{q-1}{q^{2}} B C(-1) \delta(A) \\
& -\frac{q-1}{q^{2}} A C(-1) \delta(B)-\frac{q-1}{q^{2}} A(-1) \delta(C \overline{A B}) \\
& +A B(-1)\left\{\begin{array}{l}
0 \quad \text { if } C \text { is not a square, } \\
\left(\begin{array}{l}
D \\
A
\end{array}\right)\left(\begin{array}{r}
B \bar{D} \\
A B \bar{D}
\end{array}\right)+\left(\begin{array}{r}
\varphi D \\
A
\end{array}\right)\left(\begin{array}{r}
\varphi B \bar{D} \\
\varphi A B \bar{D}
\end{array}\right) \quad \text { if } C=D^{2} .
\end{array}\right.
\end{aligned}
$$

Proof. Set $x=1$ in Theorem 4.28.

Theorems 4.35 and 4.37 are the analogues of Saalschütz's theorem and Dixon's theorem, respectively. The theorems of Watson and Whipple are obtained from Dixon's theorem by using the ${ }_{3} F_{2}(1)$ transformations.

Theorem 4.38. For characters $A, B$ and $C$ of $\operatorname{GF}(q)$,

(i) ${ }_{3} F_{2}\left(\begin{array}{rrr}A^{2} C, & B, & C \\ & A C, & B^{2}\end{array}\right)$

$$
\begin{aligned}
= & \frac{q-1}{q^{2}} C(-1) \delta(A)-\frac{q-1}{q^{2}} A C(-1) \delta(B)-\frac{q-1}{q^{2}} C(-1) \delta(A \bar{B} C) \\
& +A(-1)\left\{\begin{array}{l}
0 \quad \text { if } C \text { is not a square, } \\
\left(\begin{array}{l}
D \\
\bar{A}
\end{array}\right)\left(\begin{array}{r}
B \bar{D} \\
\bar{A} B \bar{D}
\end{array}\right)+\left(\begin{array}{r}
\varphi D \\
\bar{A}
\end{array}\right)\left(\begin{array}{r}
\varphi B \bar{D} \\
\varphi \bar{A} B \bar{D}
\end{array}\right) \quad \text { if } C=D^{2},
\end{array}\right.
\end{aligned}
$$

(ii)

$$
\begin{aligned}
{ }_{3} F_{2}\left(\begin{array}{rr}
A, \quad B, & \bar{B} \\
A^{2} \bar{C}, & C
\end{array}\right)= & \frac{q-1}{q^{2}} \delta(A)-\frac{q-1}{q^{2}} \delta(B)-\frac{q-1}{q^{2}} A(-1) \delta(C \bar{A}) \\
& +B(-1)\left\{\begin{array}{r}
0 \quad \text { if } B C \text { is not a square, } \\
\left(\begin{array}{l}
D \\
A
\end{array}\right)\left(\begin{array}{r}
B \bar{D} \\
A B \bar{D}
\end{array}\right)+\left(\begin{array}{r}
\varphi D \\
A
\end{array}\right)\left(\begin{array}{r}
\varphi B \bar{D} \\
\varphi A B \bar{D}
\end{array}\right) \quad \text { if } B C=D^{2} .
\end{array}\right.
\end{aligned}
$$


Proof. For (i), apply transformation (4.25) to the hypergeometric series in Theorem 4.37 and replace $A$ by $\bar{A}$. For (ii), apply transformation (4.23) and replace $C$ by $B C$.

5. Remarks. Several of the results in this paper have previously appeared in the literature under different guises. For example, the result [11, Theorem 2; 15, The.orem A2],

$$
\begin{aligned}
& \frac{1}{q-1} \sum_{\chi} G(A \chi) G(B \bar{\chi}) G(C \bar{\chi}) G(D \bar{\chi}) \\
& \quad=\frac{G(A B) G(A D) G(B C) G(C D)}{G(A B C D)}+q(q-1) A C(-1) \delta(A B C D)
\end{aligned}
$$

is equivalent to Theorem 4.9 via (2.9). Also, results [5, (4)] and [5, (5)] are essentially two different formulations of Dixon's theorem, Theorem 4.37.

It is the author's hope that techniques involving hypergeometric series over finite fields will provide a useful and elementary set of tools for dealing with problems related to character sums. As an example, see [10] in which the character sum

$$
\sum_{x, y} \varphi(x y(1+x)(1+y)(x+y))
$$

is evaluated over $\operatorname{GF}(P)$. This evaluation can be used to obtain an explicit count of the number of points on the surface $\left(x^{2}+1\right)\left(y^{2}+1\right)\left(x^{2}+y^{2}\right)=z^{2}$ over $\operatorname{GF}(P)$ and confirms a conjecture of Evans [8, p. 370].

Even the binomial theorem, Theorem 2.3, can be quite useful. For example, the Jacobsthal sum, $H_{n}(a)$, in our notation is defined by

$$
H_{n}(a)=\sum_{x} \varphi(x) \varphi\left(a+x^{n}\right) .
$$

A classical result involving the Jacobsthal sum is [16, p. 231]

$$
H_{n}(a)=\varphi(a) \psi(-1) \sum_{j=0}^{d-1} \psi^{2 j+1}(a) J\left(\psi^{2 j+1}, \varphi\right),
$$

where $d=\operatorname{gcd}(n, q-1)$ and $\psi$ is a character of order $2 d$. This result is easily proved by elementary techniques but it follows almost immediately from orthogonality and the binomial theorem. We have

$$
\begin{aligned}
\sum_{x} \varphi(x) \varphi\left(a+x^{n}\right) & =\varphi(a) \sum_{x} \varphi(x) \varphi\left(1+\frac{x^{n}}{a}\right) \\
& =\frac{\varphi(a)}{q-1} \sum_{x, \chi} J(\varphi, \bar{\chi}) \bar{\chi}(-a) \varphi \chi^{n}(x)
\end{aligned}
$$

by Theorem 2.3. By orthogonality, the $x$-sum is 0 unless $\chi$ is an odd power of $\psi$, which establishes the result. 
The theory presented in this paper is by no means complete. Three notable transformations for which analogues have not been given are the balanced ${ }_{4} F_{3}(1)$ transformation [2, p. 56]

$$
\begin{aligned}
& { }_{4} F_{3}\left(\begin{array}{cccc}
a, & b, & c, & -n \\
& e, & f, & g
\end{array} \mid 1\right)
\end{aligned}
$$

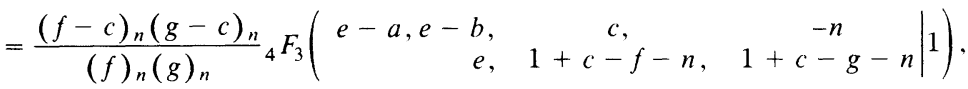

provided $e+f+g=1-n+a+b+c$, the transformation of a nearly poised ${ }_{4} F_{3}(1)$ to a 1 -balanced special ${ }_{5} F_{4}(1)[2$, p. 33]

$$
\begin{aligned}
& { }_{4} F_{3}\left(\begin{array}{cccc}
a, & b, & c, & -m \\
& 1+a-b, & 1+a-c, & w
\end{array} \mid 1\right)
\end{aligned}
$$

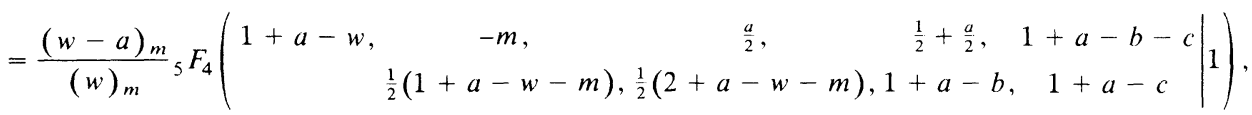

and Whipple's transformation of a very well poised ${ }_{7} F_{6}(1)$ to a 1-balanced ${ }_{4} F_{3}(1)[2$, p. 25]

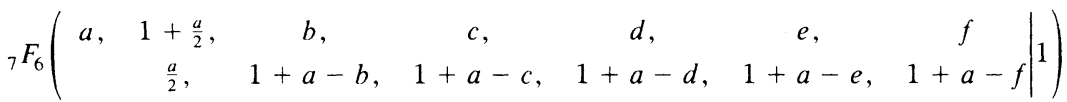

$$
\begin{aligned}
& =\frac{\Gamma(1+a-d) \Gamma(1+a-e) \Gamma(1+a-f) \Gamma(1+a-d-e-f)}{\Gamma(1+a) \Gamma(1+a-e-f) \Gamma(1+a-d-f) \Gamma(1+a-d-e)}
\end{aligned}
$$

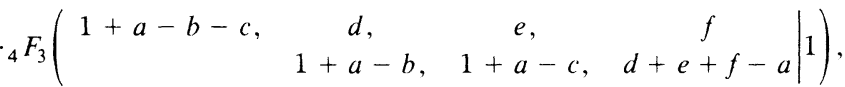

provided the left-hand side converges and the right-hand side terminates. Two evaluation theorems for which analogues have not been given are the evaluation of a very well poised ${ }_{5} F_{4}(1)[17$, p. 56]

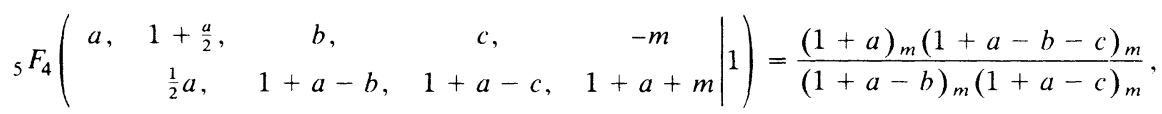

and Dougall's theorem [17, p. 56]

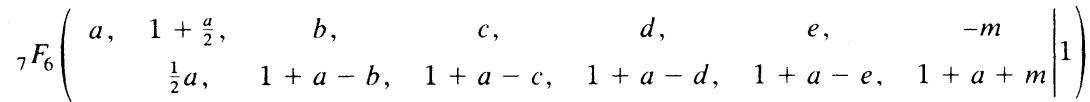

$$
\begin{aligned}
& =\frac{(1+a)_{m}(1+a-b-c)_{m}(1+a-b-d)_{m}(1+a-c-d)_{m}}{(1+a-b)_{m}(1+a-c)_{m}(1+a-d)_{m}(1+a-b-c-d)_{m}},
\end{aligned}
$$

provided $1+2 a=b+c+d+e-m$.

Analogues for the results in (5.4) and (5.5) are easily accessible. To obtain an analogue for (5.5) multiply both sides of the quadratic transformation, Theorem 4.28 , by $(M W(-1) / q) \bar{M}(x) M W(1-x)$ and sum on $x$. If the series involved are 
nondegenerate, the resulting expression is

$$
\begin{aligned}
& { }_{4} F_{3}\left(\begin{array}{cccc}
A, & B, & C, & \bar{M} \\
& C \bar{A}, & C \bar{B}, & W
\end{array} \mid 1\right)=\frac{A B C W(-1)}{q^{2}} M W(2)+\frac{B C W(-1)}{q}\left(\begin{array}{c}
B \\
A B
\end{array}\right)\left(\begin{array}{c}
M W C \\
W
\end{array}\right) \\
& +B(-1) \frac{q}{q-1} \sum_{\chi}\left(\begin{array}{c}
C \chi^{2} \\
\chi
\end{array}\right)\left(\begin{array}{c}
C \chi \\
\bar{A} C \chi
\end{array}\right)\left(\begin{array}{c}
\overline{A B} C \chi \\
\bar{B} C \chi
\end{array}\right)\left(\begin{array}{c}
\bar{M} \chi \\
\overline{M W} C \chi^{2}
\end{array}\right) \chi(-1) .
\end{aligned}
$$

The series on the right-hand side of (5.9) is the best analogue available for

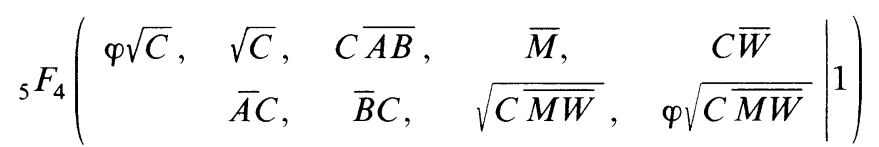

if neither $C$ nor $C \overline{M W}$ is a square. To obtain an analogue for (5.4), multiply two transformations of type 4.4(iv) to obtain

$$
\begin{aligned}
{ }_{2} F_{1}\left(\begin{array}{r}
A, B \\
C
\end{array} \mid x\right)_{2} F_{1}\left(\begin{array}{r}
D, E \\
F
\end{array} \mid x\right)=A D(-1)\left(\begin{array}{r}
B \\
\bar{A} C
\end{array}\right)\left(\begin{array}{r}
E \\
\bar{D} F
\end{array}\right) \delta(1-x) \\
+C F(-1) F C \overline{A B D E}(1-x)_{2} F_{1}\left(\begin{array}{r}
C \bar{A}, C \bar{B} \\
C
\end{array} \mid x\right){ }_{2} F_{1}\left(\begin{array}{r}
F \bar{D}, F \bar{E} \\
F
\end{array}\right) .
\end{aligned}
$$

If we pick the parameters so that $F C \overline{A B D E}(1-x)=\varepsilon(1-x)=1-\delta(1-x)$, then equating coefficients of $\bar{G}(x)$ gives

$$
\begin{aligned}
& \operatorname{DEF}(-1) \frac{q}{q-1}{ }_{4} F_{3}\left(\begin{array}{cccc}
A, & B, & G, & \bar{F} G \\
& C, & \bar{D} G, & \bar{E} G
\end{array}\right) \\
& =C D E(-1) \frac{q}{q-1}{ }_{4} F_{3}\left(\begin{array}{cccc}
C \bar{A}, & C \bar{B}, & G, & G \bar{F} \\
C, & G D \bar{F}, & G E \bar{F}
\end{array} \mid 1\right) \\
& +\frac{A D(-1)}{q-1}\left(\begin{array}{r}
B \\
\overline{A C}
\end{array}\right)\left(\begin{array}{r}
E \\
\bar{D} F
\end{array}\right)-\frac{A D(-1)}{q-1}\left(\begin{array}{r}
C \bar{B} \\
A
\end{array}\right)\left(\begin{array}{r}
F \bar{E} \\
D
\end{array}\right) \text {, }
\end{aligned}
$$

or after relabeling the parameters,

$$
\begin{aligned}
& { }_{4} F_{3}\left(\begin{array}{cccc}
A, & B, & C, & D \\
E, & F, & G
\end{array} \mid 1\right)=\frac{A D G(-1)}{q}\left(\begin{array}{r}
B \\
\overline{A E}
\end{array}\right)\left(\begin{array}{c}
C \bar{G} \\
\bar{D} F
\end{array}\right)-\frac{A D G(-1)}{q}\left(\begin{array}{r}
E \bar{B} \\
A
\end{array}\right)\left(\begin{array}{c}
\bar{D} G \\
C \bar{F}
\end{array}\right)
\end{aligned}
$$

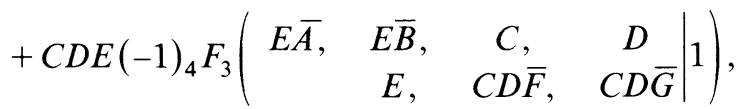

provided $A B C D=E F G$. These techniques do not seem to be good enough to give analogues for (5.6)-(5.8) and, in fact, there is no known analogue for these results.

\section{REFERENCES}

1. R. Askey, Orthogonal polynomials and special functions, SIAM, Philadelphia, Pa., 1975.

2. W. Bailey, Generalized hypergeometric series, Cambridge Univ. Press, Cambridge, 1935.

3. H. Davenpört and H. Hasse, Die Nullstellen der Kongruenzzetafunktionen in gewissen zyklischen Fällen, J. Reine Angew. Math. 172 (1934), 151-182.

4. A. Erdélyi, Higher transcendental functions, Vol. 1, McGraw-Hill, New York, 1935. 
5. R. Evans, Identities for products of Gauss sums over finite fields, Enseignement Math. 27 (1981), 197-209.

6. __ Character sum analogues of constant term identities for root systems, Israel J. Math. 46 (1983), 189-196.

7. __ Hermite character sums, Pacific J. Math. 122 (1986), 357-390.

8. R. Evans. J. Pulham and J. Sheehan, On the number of complete subgraphs contained in certain graphs, J. Combin. Theory Ser. B 30 (1981), 364-371.

9. J. Greene, Character sum analogues for hypergeometric and generalized hypergeometric functions over finite fields, Ph.D. thesis, Univ. of Minnesota, Minneapolis, 1984.

10. J. Greene and D. Stanton, A character sum evaluation and Gaussian hypergeometric series, J. Number Theory 23 (1986), 136-148.

11. A. Helversen-Pasotto, L'identité de Barnes pour les corps finis, C.R. Acad. Sci. Paris Sér. A 286 (1978), 297-300.

12. K. Ireland and M. Rosen, A Classical introduction to modern number theory, Springer-Verlag, New York, 1982.

13. C. Jacobi, Über die reisteilung und ihre Anwendung auf die Zahlentheorie, J. Reine Angew. Math. 30 (1846), 166-182.

14. N. Koblitz, The number of points on certain families of hypersurfaces over finite fields, Compositio Math. 48 (1983), 3-23.

15. W. Li and J. Soto-Andrade, Barnes' identities and representations of GL(2). Part I: Finite field case, J. Reine Angew Math. 344 (1983), 171-179.

16. R. Lidl and H. Niederreiter, Finite fields, Encyclopedia of Mathematics and Its Applications (G.-C. Rota, ed.), Vol. 20, Addison-Wesley, Cambridge, Mass., 1983.

17. L. Slater, Generalized hypergeometric functions, Cambridge Univ. Press, Cambridge, 1966.

18. E. Whittaker and G. Watson, Modern analysis, Cambridge Univ. Press, Cambridge, 1947.

Department of Mathematics, Southern Illinois University, Carbondale, Illinois 62901 